Conclusions Personality and autonomic neurophysiology influences subcortical morphological changes identified in PCs. Future research should investigate associations of endophenotypic characteristics to brain structure and function in health and chronic visceral pain to establish biomarkers for personalised medicine-based approaches.

\section{PWE-147 SYSTEMATIC STUDY OF GASTROINTESTINAL SYMPTOMS AND QUALITY OF LIFE IN CYSTIC FIBROSIS: THE 'CF GUT'}

\author{
${ }^{1}$ Bu'Hussain Hayee*, ${ }^{1}$ Kerry-Lee Watson, ${ }^{1}$ Emma Howard, ${ }^{1}$ Penelope Hutchings, \\ ${ }^{2}$ Sanchika Campbell, ${ }^{2}$ Anna Simpson, 'Caroline Elston. 'King's College Hospital, London, \\ UK; ${ }^{2}$ King's College London, UK
}

\subsection{6/gutjnl-2018-BSGAbstracts.441}

Introduction The 'CF gut' is a novel term encompassing the range of gastrointestinal (GI) symptoms recognised in patients with cystic fibrosis (CF). There are no accepted or validated assessment tools and neither the range nor the frequency of symptoms has been described. Evidence from our CF-GI clinic demonstrated that symptoms were not accounted for by pancreatic insufficiency (PI) and appeared to correspond to those seen in IBS. These symptoms will impact quality of life (QOL) outside of pulmonary morbidity, therefore it is not clear whether current CF-related QOL tools are sufficient.

Methods Consecutive patients attending specialist CF clinics were asked to complete questionnaires: Patient Health (PHQ9); Generalised Anxiety (GAD-7); GI symptom rating scale (GSRS); IBS symptom severity score (IBS-SSS); CF-related quality of life (CFQR). Demographics, BMI, CF genotype, PI status and enzyme replacement therapy (PERT) were recorded. Patients with pre-existing coeliac disease or inflammatory bowel disease were excluded. Questionnaires were altered to remove the term 'IBS' and patients were asked about 'GI symptoms' instead.

Results Results from the total cohort of 176 patients will be forthcoming, but we present interim data from 107 (mean age $27.8 \pm 9.6 \mathrm{y} ; 60 \mathrm{~F}$; 94 PI $(88 \%)$ of whom 2 were not taking PERT; mean BMI $22.1 \mathrm{~kg} / \mathrm{m}^{2}, \quad \mathrm{FEV}_{1} 59 \%$ predicted). 53 (49.5\%) were $\Delta$ F508 homozygous.

69/107 (65\%) met Rome IV criteria for IBS, with 47 (44\%) reporting significant symptoms (IBS-SSS >80). Using the GSRS we created a 'heatmap' to describe the range and severity of symptoms as IBS-SSS increased (figure: 'traffic light' colour chart for mild-moderate-severe, columns 1-11 are each descriptor in the GSRS; rows are individual patients with IBS-SSS increasing down left-most column).

With patients grouped as IBS-SSS $<80$ or $>80$, significant differences were observed in anxiety and depression as well as across all domains of the CFQR. There was no correlation between IBS-SSS and any CFQR domain (particularly that relating to GI symptoms), suggesting that the latter is insufficient to describe the CF gut.

Conclusion This is the first ever systematic study using validated symptom scores to describe the range of GI symptoms in CF. These do not correlate with PERT or genotype and appear to be captured well by the IBS-SSS, but not the CFQR. Further work will be aimed at establishing accurate screening and assessment tools for this phenotype. Therapeutic trials in CF may use these already validated tools to demonstrate a positive impact on 'non-respiratory' symptoms and QOL.

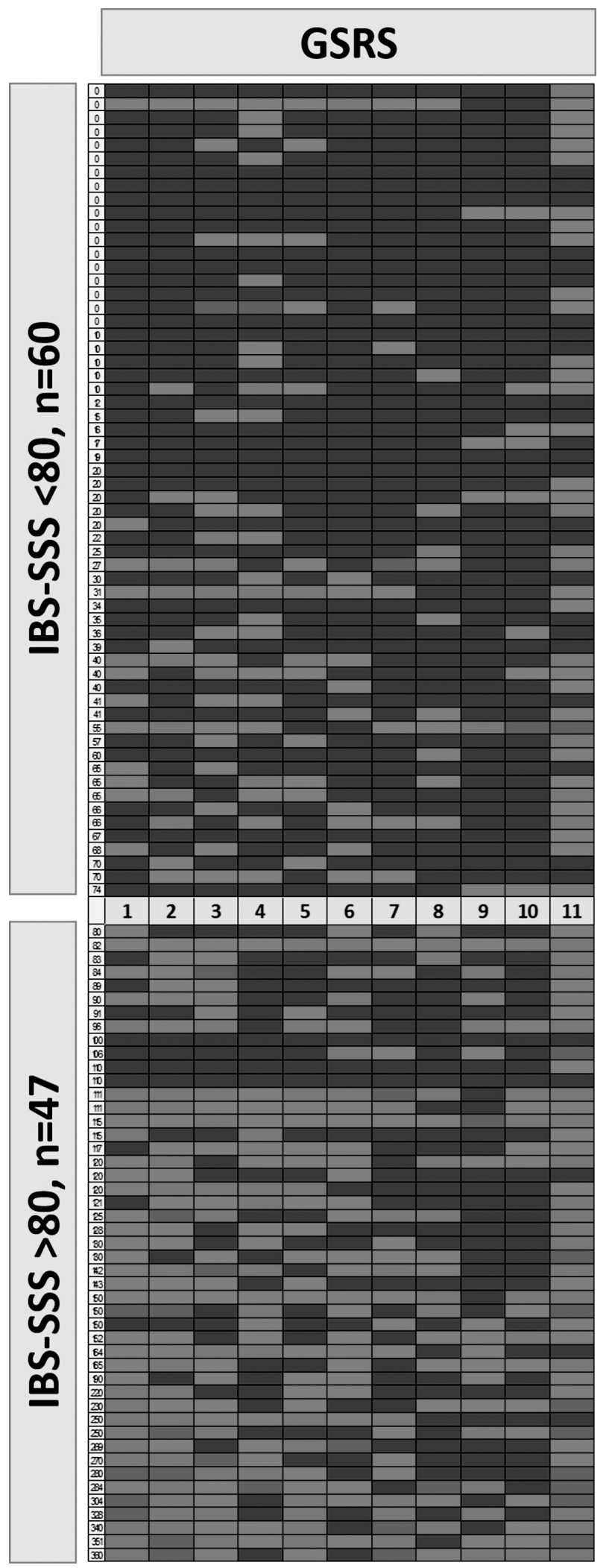

Abstract PWE-147 Figure 1 


\section{Gastroenterology Service}

\section{OTU-028 BENCHMARKING OF ACTIVITY, PROCESS AND OUTCOME OF EMERGENCY ADMISSION FOR ULCERATIVE COLITIS ACROSS ENGLISH HOSPITALS}

\author{
${ }^{1}$ Mustafa Shawihdi*, ${ }^{1}$ Susanna Dodd, ${ }^{1}$ Duncan Appelbe, ${ }^{1}$ Pete Dixon, ${ }^{1}$ Ruth Grainger, \\ ${ }^{3}$ Fraser Cummings, ${ }^{2}$ Stuart Bloom, ${ }^{1}$ Keith Bodger*. ${ }^{1}$ University of Liverpool, Liverpool, UK; \\ ${ }^{2}$ University College London Hospital, London, UK; ${ }^{3}$ University Hospitals Southampton, \\ Southampton, UK
}

\subsection{6/gutjnl-2018-BSGAbstracts.442}

Introduction The UK IBD Registry aims to make information work better for patients, clinical teams and the NHS. As part of the benchmarking reports provided to participating Trusts, we have developed organisational level metrics from routinely collected hospital episode statistics (HES) data - working with front-line teams to iteratively develop reports with feedback on content and local face-validity. We report national-level findings and institutional variation in activity, process and outcome of emergency care for UC.

Methods Admitted patient care data for English hospitals were analysed, identifying all-cause admissions for patients with UC and constructing algorithms to identify emergency activity, track process and outcome for UC-specific emergency admissions (UC-Em-Ad), including in-hospital death (I-H-D) and emergency surgery (Em-Surg), all-cause 30 day readmission (30D-RA) and twelve month outcome. Reports containing 5 year national and local trends and cumulative 5 year performance were distributed to sites in Dec 2017. This analysis summarises selected data for 133 Trusts present in all fiscal years $(11 / 12$ to $15 / 16)$.

Results Nationally, there were 31,371 UC-Em-Ad (2 65799 bed days; median LoS 6 days; 22809 patients; mean age 40 years; male 50\%; additional coded co-morbidities in $23 \%)$ with 1451 Em-Surg $(4.62 \%=$ crude surgery rate; mean age 44 years; male 56.1\%), 324 I-H-D $(1.03 \%=$ crude mortality rate; mean age 76 years; $67 \%$ had additional coded co-morbidities; only $16 \%$ of deaths were post-surgery), 4916 30D-RA $(15.7 \%=$ crude readmission rate). At Trust level, mean $(95 \%$ limits) for indirectly standardised rates were: I-H-D $1.03 \% \quad(0.90 \%-1.15 \%)$, Em-Surg $4.79 \%$ (4.31\%-5.27\%), 30D-RA 15.55\% (15.0\%-16.1\%). Few outliers were identified and none consistently over time, with no significant trends identified for volume-outcome relationships. Funnel plots and regression analyses will be presented.

Conclusions These data provide real-world insights into processes and outcomes of emergency care for UC across England in the last five years, with a series of metrics to support both national and local quality improvement efforts. Linkages between HES and local Registry data offers potential to validate, refine and extend these benchmarking metrics.

Funding Crohn's and Colitis UK.

\section{OTU-029 FAECAL IMMUNOCHEMICAL TESTS (FIT) FOR SURVEILLANCE AFTER SCREENING AND POLYPECTOMY: AN ACCURACY AND EFFICIENCY STUDY}

${ }^{1}$ Emma Robbins*, 'Kate Wooldrage, ${ }^{1}$ Eilidh MacRae, 'lain Stenson, 'Bhavita Patel, ${ }^{1}$ Kevin Pack, ${ }^{2}$ Carolyn Piggott, ${ }^{2}$ Sheena Pearson, ${ }^{2}$ Julia Snowball, ${ }^{3}$ Stephen Duffy, ${ }^{2}$ Stephen Halloran, 'Wendy Atkin, ${ }^{1}$ Amanda Cross. ${ }^{1}$ Cancer Screening and Prevention Research Group, Imperial College London, London; ${ }^{2}$ Bowel Cancer Screening Programme Southern Hub, Guildford; ${ }^{3}$ Centre for Cancer Prevention, Wolfson Institute of Preventive Medicine, Queen Mary University, London

\subsection{6/gutjnl-2018-BSGAbstracts.443}

Introduction Individuals at intermediate-risk for colorectal cancer (CRC) following adenoma removal within the English Bowel Cancer Screening Programme (BCSP) are invited for three-yearly surveillance colonoscopy. Given the invasive nature of colonoscopy and scarcity of endoscopy resources, there is a need for an alternative surveillance method. We aimed to determine whether annual testing with the faecal immunochemical test (FIT) is an effective alternative.

Methods Individuals aged 60-72 years and scheduled for surveillance following removal of intermediate-risk adenomas were recruited within the BCSP from January 2012 to December 2013. Quantitative FIT (OC-Sensor, Eiken) was offered at one, two, and three years post-polypectomy. Invitees who returned a completed consent form and an analysable FIT at Round 1 were included. Participants testing positive $(\geq 40 \mu \mathrm{g}$ haemoglobin $(\mathrm{Hb}) / \mathrm{g}$ faeces) at Rounds 1 or 2 were offered early colonoscopy and were not invited to further FIT rounds. All other participants were offered the routine three-year surveillance colonoscopy. Diagnostic accuracy for CRC and advanced adenomas (AAs: adenomas $\geq 10 \mathrm{~mm}$, with tubulovillous or villous histology, or high grade dysplasia) was calculated at each round, using colonoscopy as the reference standard. We estimated diagnostic accuracy with lower haemoglobin thresholds and multiple rounds.

Results Of 8008 invitees, 5946 (74\%) consented and returned an analysable FIT at Round 1. Uptake of FIT was higher (97\%) in Rounds 2 and 3. FIT positivity decreased by round, from $6 \%$ to $4 \%$ in Rounds 1 to 3 . In total, 26 participants were diagnosed with CRC and 443 with AAs. At $40 \mu \mathrm{g} / \mathrm{g}$, sensitivity and specificity of the first FIT were, respectively, $31 \%$ and $94 \%$ for CRC and $18 \%$ and $95 \%$ for AAs. Sensitivities for CRC and AAs were higher, and specificities lower, with lower thresholds and multiple rounds. At $10 \mu \mathrm{g} / \mathrm{g}$, the programme sensitivity and specificity of three rounds were, respectively, $85 \%$ and $71 \%$ for CRC and $57 \%$ and $73 \%$ for AAs.

Conclusions Annual low threshold FIT achieved relatively high sensitivity for CRC over three years. If this strategy replaced three-yearly surveillance colonoscopy, the number of colonoscopies could potentially be reduced by $70 \%$. However, sensitivity for AAs was limited. Further research is needed to consider the implications for clinical practice of missing CRCs and AAs with FIT-based surveillance. 


\section{OTU-030 FAECAL MICROBIOTA TRANSPLANTATION FOR RECURRENT CLOSTRIDIUM DIFFICILE INFECTION: REAL WORLD UK DATA}

${ }^{1} J o n a t h a n$ Digby-Bell*, ${ }^{2}$ Kamal Patel, ${ }^{3}$ Mohamed Mutalib, ${ }^{1}$ Peter Inving, ${ }^{1}$ Simon Goldenberg. 'Guy's and St Thomas' NHS Trust, London, UK; ${ }^{2}$ St George's Hospital, London, UK; ${ }^{3}$ Evelina Children's Hospital, London, UK

\subsection{6/gutjnl-2018-BSGAbstracts.444}

Introduction recurrent Clostridium difficile infection (rCDI) is a medically challenging condition with limited therapeutic options often resulting in repeated admissions to hospital with associated high financial and patient burden. Faecal microbiota transplantation (FMT) has been shown to be highly effective treatment in placebo-controlled trials, however little real life data exists particularly from UK centres. We report our experience in a single tertiary referral centre.

Method Data was collected prospectively from January 2015 to November 2017 for patient demographics, comorbidities, route of FMT administration, 30 day and 1 year mortality. Primary endpoint was resolution of diarrhoea without relapse 10 weeks after first FMT. Resolution of diarrhoea without relapse 10 weeks after second FMT was also recorded.

Results 35 adult and 2 paediatric patients were approved for FMT for treatment of rCDI of which 35 patients underwent the procedure. 2 patients clinically deteriorated before FMT could be performed such that FMT became inappropriate.

$27 / 35(77 \%)$ were female, with a mean age of 67 y (range 4-91), and a mean ASA grade of 2.0 (0-4). Patients had received $3.1(2-5)$ courses of antibiotics for clostridium difficile and $27(77 \%)$ were external referrals. 32 FMTs were performed via colonoscopy and 3 via nasojejunal tube.

3 patients died within 30 days of FMT (mean ASA grade 3.3) but none directly related to the FMT or C Diff. There was once further expected death 90 days after FMT. No other major side effects or safety concerns were seen.

Of the patients who survived to day $30 ; 28$ out of 32 $(87.5 \%)$ patients had cessation of diarrhoea without relapse after 10 weeks. 4 patients had recurrent diarrhoea within 10 weeks of FMT of whom 2 had a 2 nd FMT resulting in cessation of diarrhoea with no relapse after 10 weeks. The other two patients clinically deteriorated due to underlying medical conditions such that a 2nd FMT was inappropriate. All patients who survived had resolution of symptoms after their first or second transplant.

Conclusion FMT is a highly effective treatment for rCDI in the real world with resolution of symptoms and no relapse after 10 weeks achieved in $89.7 \%$ of patients undergoing 1 st FMT increasing to $100 \%$ after a 2 nd FMT.

No safety concerns were identified during the study period. The 3 deaths within 30 days of FMT highlight the comorbid population who develop rCDI and better patient selection is required to ensure appropriateness of FMT in high risk groups.

\section{OTU-031 MY-IBD PORTAL: PROGRESS AND IMPACT UPDATE}

\footnotetext{
1,2John Mclaughlin*, 'Sister Catherine Stansfield, 'Simon Lal, ${ }^{3}$ Keith Bodger, 'James Andrew Robinson. 'Salford Royal Hospitals, Salford, UK; ${ }^{2}$ University of Manchester, Manchester, UK; ${ }^{3}$ University of Liverpool, Liverpool, UK
}

\subsection{6/gutjnl-2018-BSGAbstracts.445}

Introduction Patients with Inflammatory Bowel Disease (IBD) often struggle with access to prompt advice regarding and place a heavy reliance on overstretched services. We have a track record in guided self-management of IBD so explored whether this could be built into a novel patient-centred portal with direct on line access to a fully personalised health record, integrating individualised plans of care and disease monitoring tools.

Methods In a unique collaboration with local patients, the patient charity Crohn's and Colitis UK, health care and IT teams, we developed, implemented and evaluated a web based portal at Salford Royal. At the outset, a user group of patients was established and their views and needs were central to each step of the development, refinement and evaluation of the portal. From this group it was clear that there was a particular demand by IBD patients to have access to their health records and reliable information.

Results We have built and refined the 'MY IBD' portal, which is fully integrated with the electronic patient record and delivers:

1. Access to personalised information: diagnosis, visual aids and links to CCUK information resources

2. Immediate access to blood results, investigation results, clinic letters

3. Disease activity assessment tools

4. A personalised plan of care available online for the patient

5. Improved communication with the IBD team: messaging facility and trigger emails when disease activity scores are high.

720 patients are now using the portal. Overall usability was scored as excellent, showing patients were helped with decision-making. Improvements were seen in perceived support $(\mathrm{p}=0.06)$ compared to non-users with a trend to improved disease related knowledge $(p=0.14)$. An average of clinic 2.9 attendances per year (2014-15) reduced to 0.6 attendances for portal self-management users (2016-2017) releasing over 500 clinic appointments. Satisfaction with self-management remains high with $98 \%$ of patients rating the process as good/ excellent. User group sessions have captured value to patients. They expressed it had 'completely changed my care', 'having up to date information about your condition leads to less stress and better health' and 'puts patients back in control of their illness'.

The portal has also been refined for integration with the IBD registry.

Conclusions Patient experience and service have improved. Portal patients are more in control, with greater independence to self-manage through better understanding of their condition. Other Trusts are now seeking to adopt the portal and we welcome enquiries to establish it in other NHS sites.

Acknowledgments funded by CCUK, 'Living with IBD'.

\section{OTU-032 INTEGRATED IBS CARE PATHWAY WITH DIRECT- ACCESS, DIETETIC-LED SERVICE - IMPACT ON PATIENT INVESTIGATION AND OUTCOMES}

Claire Oldale*, Phoebe Hodges, Samuel Waternan, Alexandra Di Mambro. Glos Hospitals NHS Foundation Trust, Cheltenham, UK

10.1136/gutjnl-2018-BSGAbstracts.446

Introduction An integrated care pathway with direct access to a dietitian-led refractory IBS (RIBS) service was set up in Gloucestershire in 2016. GPs may refer patients $<45$ years with symptoms fulfilling ROME criteria for IBS which is refractory to first 